\section{Custo e economia da prática do aleitamento materno para a família}

\section{Costs and savings for the family as the result of breast feeding}

Maria de Fátima Moura de Araújo 1

Adriana Del Fiaco 2

Letícia Silva Pimentel 3

Bethsáida de Abreu Soares Schmitz 4

1 Fundação de Ensino e Pesquisa em Ciências da Saúde e Ministério da Saúde. Av. W3 Norte, Quadra 501 Bloco A - FEPECS.

Brasília (DF), Brasil. CEP: 70.710-907.

2-4 Departamento de Nutrição. Faculdade de Ciências da Saúde.

Universidade de Brasília. Brasília (DF), Brasil.

\begin{abstract}
Objectives: to compare supplementary food cost of nursing mothers related to breast feeding with breast milk substitutes.

Methods: the cost of energy spent during lactation was calculated with the addition of one or two types of food of the usual diet to supply the extra demand of a nursing baby. Menus were designed and calculated for the mothers and food cost estimated as well as the cost of baby formulas and type C cow milk for children under six months of age according to nutritional recommendations for this phase of life considering a six month consumption period. Percentage of minimum wage spent with each type of food was determined.

Results: supplementary food offered to mothers has an average cost of $8.7 \%$ of the minimum wage. Approximately $35 \%$ of the minimum wage is spent in feeding the baby with formulas and $11 \%$ with type $C$ cow milk.

Conclusions: these values, similar to other surveys indicate the supplementary food aiming at promoting breast feeding is more cost-effective than maternal milk substitutes. The results indicate that it is cheaper and more efficient to assure supplementary food to poor nursing mothers to promote breast feeding than to distribute formulas or milk that induce early weaning.
\end{abstract}

Key words Economics, Breast feeding, Supplementary feeding

\section{Resumo}

Objetivos: comparar o custo da alimentação complementar da nutriz em relação à alimentação do bebê com substitutos do leite materno.

Métodos: o custo do gasto energético da lactação foi calculado com a adição de um ou dois alimentos da dieta habitual para suprir a demanda extra da lactante. Foram elaborados e calculados cardápios adicionais para a nutriz, e estimado o custo da alimentação com fórmula infantil e com leite de vaca tipo $C$ para lactentes menores de seis meses, segundo recomendações nutricionais para essas fases da vida, para um consumo de seis meses. Determinou-se a porcentagem de salário mínimo gasto com cada tipo de alimentação.

Resultados: observou-se que a alimentação complementar da nutriz custa em média 8,7\% do salário mínimo. Gasta-se aproximadamente $35 \%$ do salário mínimo na alimentação do bebê com fórmula infantil e $11 \%$ com leite de vaca tipo $C$.

Conclusões: os resultados apontam que é mais barato e eficaz garantir a complementação alimentar de nutrizes carentes, para se promover a amamentação, que distribuir fórmulas ou leites, e favorecer o desmame precoce.

Palavras-chave Economia, Aleitamento materno, Suplementação alimentar 


\section{Introdução}

Vários estudos têm comprovado os benefícios da amamentação natural na saúde da criança, na saúde da mulher, no fortalecimento do vínculo afetivo entre mãe e filho, bem como na economia para famílias, instituições de saúde, governos e nações, entre outros aspectos. ${ }^{1-8}$

Os benefícios econômicos do aleitamento materno são diretos, quando se compara o baixo custo da amamentação com a utilização dos substitutos do leite materno, e indiretos, quando gastos com doenças relacionadas ao aleitamento artificial são relacionados. $7,9,10$

Infelizmente, ainda é baixa no Brasil a prevalência de lactentes amamentados exclusivamente até os seis meses de idade, período recomendado pela Organização Mundial de Saúde (OMS) e pelo governo brasileiro. A Pesquisa Nacional de Prevalência de Aleitamento Materno nas Capitais Brasileiras e no Distrito Federal, 11 realizada em 1999, demonstrou que na faixa etária de 151 a 180 dias apenas 9,7 dos lactentes estavam em aleitamento materno exclusivo, enquanto que na faixa etária de 271 a 364 dias apenas $44,2 \%$ das crianças estudadas continuavam recebendo o leite materno. Em relação à alimentação complementar oportuna - que informa a proporção de lactentes com idades de 180 a 299 dias, que além do leite materno estão recebendo alimentos sólidos e semi-sólidos - apenas 48,9\% das crianças estudadas apresentaram alimentação complementar oportuna no Brasil, indicando que mais da metade já estão recebendo fórmula infantil ou outro tipo de leite nessa faixa etária.11-13

Em uma situação de impossibilidade de a criança receber leite materno, a fórmula infantil tem sido indicada, por ser modificada especialmente para atender as necessidades nutricionais e as condições fisiológicas do lactente no primeiro ano de vida. No entanto, é importante considerar que os produtos industrializados destinados a lactentes não apresentam dois benefícios fundamentais, só supridos pelo leite materno: o imunológico e o emocional. 14

Contudo, o leite de vaca, apesar de não ser a melhor escolha do ponto de vista nutricional, é a fonte mais comumente utilizada para crianças menores de um ano de idade como substituto do leite materno, pois as fórmulas infantis são substancialmente mais caras. 8

Um dos aspectos que deve ser considerado ao se orientar a alimentação de um lactente não amamentado ao peito é a condição socioeconômica da família. Muitas vezes as mães de baixo poder aquisitivo oferecem à criança uma mamadeira excessiva- mente diluída, e com elevada possibilidade de contaminação durante a reconstituição do leite, pela má qualidade da água e má higienização da mamadeira e bico. Isto expõe a criança ao risco de infecções intestinais repetidas, resultando quase sempre em desnutrição. ${ }^{14}$

Por outro lado, o único custo da amamentação exclusiva é a produção do leite materno, que é igual ao conteúdo energético do leite secretado, adicionado da energia necessária para produzi-lo.

Portanto, o custo da lactação pode ser calculado como o custo adicional de uma fração de um ou dois alimentos da dieta cotidiana, que supram a demanda aumentada de nutrientes para a mãe, 15 e que sejam economicamente acessível às famílias de baixa renda.

O presente estudo, realizado em Brasília, DF, Brasil, tem como objetivo comparar o custo da alimentação complementar da nutriz com o custo da alimentação de bebês com substitutos do leite materno, a fim de avaliar as evidências de que, em comunidades carentes, é melhor garantir a complementação alimentar da lactante, do que distribuir leite ou fórmula para crianças em fase de amamentação.

\section{Métodos}

Foram elaborados e calculados dez cardápios adicionais para uma nutriz padrão, entre 18 e 30 anos (que abrange maior número de mulheres em idade reprodutiva), peso corpóreo de $54 \mathrm{Kg}$ e $160 \mathrm{~cm}$ de altura, obedecendo às recomendações nutricionais da OMS, para essa fase da vida. 15 Para o planejamento dos cardápios foram observados alguns alimentos de maior consumo na população brasileira, segundo o "Estudo multicêntrico de consumo alimentar". 16

Para o cálculo do custo dos cardápios, pesquisaram-se os preços dos gêneros alimentícios em quatro grandes supermercados de Brasília, utilizando-se os alimentos de menor preço e determinandose a média aritmética desses. O preço foi multiplicado para um consumo de seis meses, e então calculada a porcentagem do salário mínimo, utilizada para esse gasto adicional na alimentação da nutriz.

Para o cálculo do valor econômico do aleitamento artificial com as fórmulas infantis e com o leite de vaca tipo $\mathrm{C}$, utilizaram-se as quantidades desses produtos capazes de atender às recomendações energéticas de crianças menores de seis meses de idade. Para o cálculo do custo das fórmulas infantis, considerouse que uma lata de $500 \mathrm{~g}$ de fórmula infantil é suficiente para alimentar um lactente menor de seis meses de idade por três a quatro dias, sendo necessárias, portanto, 44 latas para alimentar o bebê 
durante seis meses. ${ }^{17}$

Foram avaliadas duas fórmulas infantis de maior consumo aqui chamadas de fórmula $\mathrm{A}$ e fórmula $\mathrm{B}$, sendo o preço das mesmas pesquisado em duas farmácias e em dois grandes supermercados de Brasília, resultando em uma média aritmética de preços. Os valores foram multiplicados para um consumo de seis meses, e foram calculadas as porcentagens do salário mínimo gastas com a alimentação do bebê com fórmula artificial e com leite de vaca tipo $\mathrm{C}$, conforme exemplo do Quadro 1.17

\section{Quadro 1}

Porcentagem de salário necessária para alimentar um lactente com substitutos do leite materno durante seis meses.

Cálculo

Marca da fórmula infantil

Preço de 1 lata de $500 \mathrm{~g}$ da fórmula infantil

Preço de 44 latas de $500 \mathrm{~g}$ da fórmula infantil

Salário mínimo: 1 mês

6 meses

Custo de 44 latas de fórmula infantil de $500 \mathrm{~g}$

Salário mínimo para 6 meses

$x 100=$ $\%$

Alimentar um bebê com leite

custa:

\% do salário mínimo

Adaptado de: OMS (Organização Mundial da Saúde), UNICEF (Fundo das Nações Unidas para a Infância). Aconselhamento em aleitamento materno: curso de capacitação: guia do treinador. São Paulo; 199718

Para efeito de comparação com estudos feitos em outros países, os valores médios obtidos em reais foram convertidos para dólares americanos e calculados em valores equivalentes a um ano de alimentação, tanto da mãe quanto da criança. Realizou-se, então, uma análise comparativa dos custos da alimentação complementar da nutriz em relação ao uso de fórmulas artificiais e leite de vaca, na nutrição do lactente.

\section{Resultados}

Os cardápios sugeridos encontram-se na Tabela 1, e atendem, em média, às necessidades de calorias, proteínas, ${ }^{15}$ cálcio, vitaminas $\mathrm{A}$ e $\mathrm{C}$ recomendadas pela Recommended Dietary Allowances (RDA) para a lactante nos primeiros seis meses, tendo sido utilizados alimentos de uso comum à população.
Os percentuais do salário mínimo encontrados para os diferentes tipos de gastos com alimentação podem ser visualizados também na Tabela 1 , tendo sido verificados os custos da alimentação da mãe com base no custo médio dos cardápios sugeridos, além do custo da alimentação do bebê com fórmula infantil e leite tipo $\mathrm{C}$.

Observou-se que alimentar uma nutriz por seis meses com os cardápios adicionais custaria, em média, o correspondente a $8,7 \%$ do salário mínimo acumulado nesse período.

Apesar de alguns cardápios isoladamente não fornecerem a quantidade necessária de todos os nutrientes analisados, e determinados alimentos sugeridos não apresentarem proteína de alto valor biológico, a variação desses cardápios ao longo dos dias supre a demanda adicional da nutriz, como pode ser visto pelos valores médios dos mesmos, e representa a realidade da população. 
Tabela 1

Cardápios complementares sugeridos para a lactante.

\begin{tabular}{|c|c|c|c|c|c|c|}
\hline Alimentos & Quantidade & Calorias & Proteínas (g) & Vitamina A (mcg) & Vitamina C (mg) & Cálcio (mg) \\
\hline Arroz & $160 \mathrm{~g}$ & & & & & \\
\hline Feijão & $80 \mathrm{~g}$ & $520 \mathrm{Kcal}$ & 23,12 & 46,48 & 0,88 & 565,60 \\
\hline Leite C & $400 \mathrm{ml}$ & & & & & \\
\hline Arroz & $160 \mathrm{~g}$ & & & & & \\
\hline Feijão & $80 \mathrm{~g}$ & $321 \mathrm{Kcal}$ & 10,78 & 2812,50 & 10,18 & 99,90 \\
\hline Cenoura & $100 \mathrm{~g}$ & & & & & \\
\hline Arroz & $160 \mathrm{~g}$ & & & & & \\
\hline Feijão & $80 \mathrm{~g}$ & $462 \mathrm{Kcal}$ & 13,52 & 89,24 & 213,68 & 73,60 \\
\hline Laranja & $400 \mathrm{ml}$ & & & & & \\
\hline Leite C & $400 \mathrm{ml}$ & & & 48,48 & & \\
\hline Beterraba & $100 \mathrm{~g}$ & $570 \mathrm{Kcal}$ & 24,48 & & 5,00 & 405,00 \\
\hline Pão francês & $100 \mathrm{~g}$ & & & & & \\
\hline Leite C & $400 \mathrm{ml}$ & & & & & \\
\hline Banana & $106 \mathrm{~g}$ & $480 \mathrm{Kcal}$ & 19,44 & 57,08 & 14,84 & 351,40 \\
\hline Pão francês & $50 \mathrm{~g}$ & & & & & \\
\hline Arroz & $160 \mathrm{~g}$ & & & & & \\
\hline Feijão & $80 \mathrm{~g}$ & $429 \mathrm{Kcal}$ & 31,26 & 4,00 & 0,88 & 85,60 \\
\hline Carne magra & $100 \mathrm{~g}$ & & & & & \\
\hline Arroz & $160 \mathrm{~g}$ & & & & & \\
\hline Banana & $106 \mathrm{~g}$ & $366 \mathrm{Kcal}$ & 17,78 & 13,00 & 15,72 & 56,54 \\
\hline Carne magra & $60 \mathrm{~g}$ & & & & & \\
\hline Abóbora & $100 \mathrm{~g}$ & & & & & \\
\hline Cenoura & $400 \mathrm{~g}$ & $459 \mathrm{Kcal}$ & 18,20 & 2292,52 & 217,56 & 519,50 \\
\hline Leite C & $400 \mathrm{ml}$ & & & & & \\
\hline Banana & $106 \mathrm{~g}$ & & & & & \\
\hline Cenoura & $100 \mathrm{~g}$ & $423 \mathrm{Kcal}$ & 11,81 & 2823,10 & 24,14 & 153,20 \\
\hline Pão francês & $100 \mathrm{~g}$ & & & & & \\
\hline Arroz & $160 \mathrm{~g}$ & & & & & \\
\hline Feijão & $80 \mathrm{~g}$ & $564 \mathrm{Kcal}$ & 19,18 & - & 0,88 & 184,60 \\
\hline Pão francês & $100 \mathrm{~g}$ & & & & & \\
\hline Média & - & $460 \mathrm{Kcal}$ & 18,95 & 815,64 & 50,37 & 249,49 \\
\hline RDA & - & $+500 \mathrm{Kcal}$ & $+16 \mathrm{~g}$ & $+500 \mathrm{mcg}$ & $+35 \mathrm{mg}$ & $+400 \mathrm{~g}$ \\
\hline
\end{tabular}

RDA = Recommended Dietary Allowances.

Fonte: National Research Council. Recommended Dietary Allowances. 10th ed. Washington (DC): National Academy; 1989. 
A análise do custo envolvido na manutenção de um lactente com fórmulas infantis, utilizando-se para tal a observação do custo de dois tipos de fórmulas (A e B), indicou que alimentar um bebê com a fórmula $\mathrm{A}$, corresponderia a $36 \%$ do salário mínimo acumulado em um período de seis meses, e, para a fórmula B, o percentual corresponderia a $34 \%$. Obteve-se assim uma média correspondendo a $35 \%$ do salário mínimo, expressa na Figura 1.

Quanto à análise do custo referente à utilização do leite tipo C, observou-se que o valor necessário à manutenção de um lactente por seis meses seria o equivalente a $11 \%$ do salário mínimo acumulado em um período de seis meses.

Foi realizada também uma análise comparativa dos valores médios em dólares, equivalentes ao custo da alimentação complementar da nutriz e do gasto com alimentação artificial da criança por um ano, buscando-se uma comparação com outros estudos, o que pode ser visualizado na Figura 2.

\section{Figura 1}

Percentual do salário mínimo gasto segundo tipo de alimentação. Distrito Federal (DF), 2000.

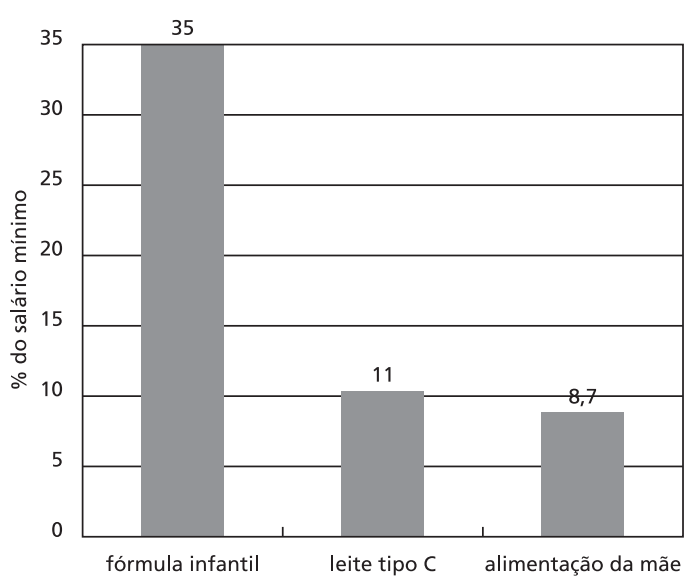

Fonte: Brasil. Pesquisa de campo. Distrito Federal (DF); 2000

\section{Figura 2}

Custo anual em dólares da alimentação complementar da nutriz e da alimentação de bebês com substitutos do leite materno.

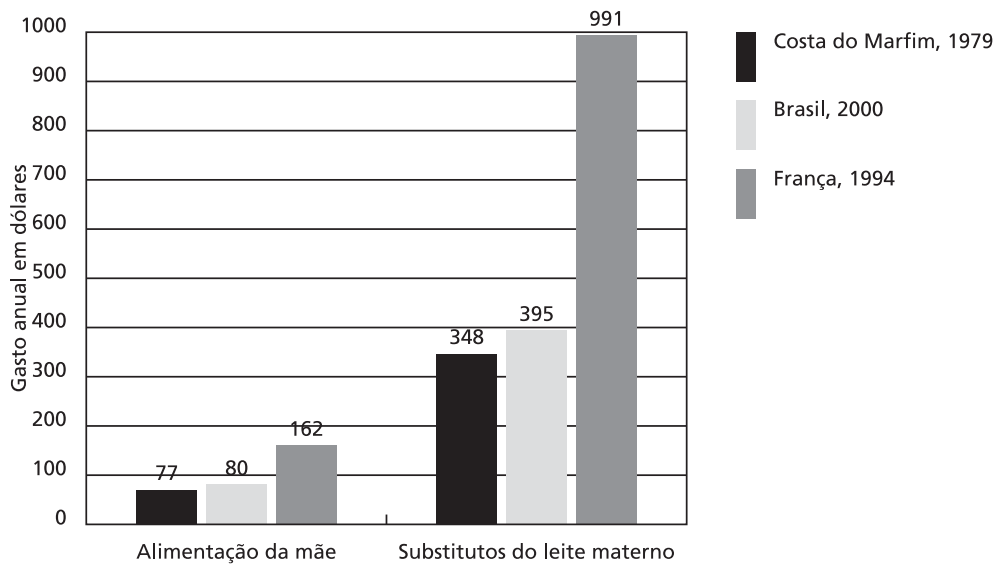

Fontes: Brasil: Pesquisa de campo. Brasília (DF); 2000.

Fonte para infomações sobre Costa do Marfim e França. WABA (World Alliance for Breastfeeding Action). World Breastfeeding week. Disponível em URL: http://www.waba.org.br/folder98.htm. ${ }^{19}$ 


\section{Discussão}

O valor econômico da amamentação, tanto para a família como para governos, em seus diferentes níveis de gestão, deve ser considerado. Incluir o leite humano nos cálculos orçamentários pode provar seu valor econômico, e, dessa forma, demonstrar o real peso dessa contribuição em termos alimentares para a nação.

Nos Estados Unidos, os programas materno-infantis gastam o dobro por mãe que faz uso de substitutos artificiais do leite materno para seus filhos, em relação aos gastos com alimentos para as mães que amamentam. Para cada bebê amamentado durante seis meses, o governo dos EUA pode economizar de US $\$ 450$ a US $\$ 800$ em custos de saúde e bem-estar social. 19

O custo do uso de leite artificial é especialmente oneroso ao se tratar de países em desenvolvimento, uma vez que, nos países do primeiro mundo, o leite faz parte da dieta diária usual, e é um alimento relativamente acessível. Por outro lado, nos demais países, o leite pode ser um item de luxo. Como exemplo, observa-se que na Inglaterra o custo do uso do leite artificial é aproximadamente $2 \%$ do salário mínimo, enquanto que em países em desenvolvimento esse valor pode variar entre 10 e $80 \%$ do salário mínimo. 19

$\mathrm{O}$ alto custo do leite artificial leva a um comportamento já comprovado, que é o da diluição do leite, com graves conseqüências para o crescimento, desenvolvimento e a própria sobrevivência das crianças. É difícil medir em termos puramente econômicos os itens relacionados aos cuidados com a criança alimentada artificialmente, e sua maior exposição às infecções. Entretanto, estimativas conservadoras colocam este valor em termos de bilhões de dólares a cada ano, montante este que poderia ser investido em medidas preventivas. 20,21

No presente estudo, comparando-se o custo da alimentação complementar da nutriz com o custo de fórmulas infantis ou leite de vaca para a nutrição do bebê, por seis meses, verifica-se que acrescentar na dieta da mãe alimentos do seu consumo habitual significa, em média, 8,7\% do salário mínimo acumulado nesse período. Em contrapartida, gastam-se aproximadamente $35 \%$ do salário mínimo na alimentação do bebê com fórmulas infantis e $11 \%$ com leite tipo $\mathrm{C}$.

Chama a atenção o fato de que não estão computados os gastos adicionais com bicos e mamadeiras, combustível para ferver a água e esterilizar mamadeiras, além de possíveis gastos em saúde com o uso de medicamentos, combustível e tempo para se deslocar ao hospital, decorrentes das morbidades associadas com maior freqüência aos lactentes alimentados artificialmente.

Observa-se, portanto, que complementar a alimentação da nutriz é mais econômico para a família e para o Estado, do que substituir o leite materno por leite de vaca ou fórmula artificial na alimentação do bebê.

São raros os estudos que comparam o custo da alimentação complementar da mãe com o uso de substitutos do leite materno. Visando uma análise comparativa dos dados obtidos no Brasil com outros trabalhos, e considerando que o momento de sua obtenção dos mesmos era diferente, procurou-se fazer essa análise tomando como referência os valores anuais em dólares. Observou-se que os resultados deste estudo foram similares aos observados na Costa do Marfim e na França ainda que em épocas diferentes. $7,8,17$

A redução nas ações de incentivo ao aleitamento materno pode levar ao decréscimo dos índices de aleitamento materno e conseqüentemente, a um maior gasto com substitutos do leite materno e com a assistência à saúde em nível nacional, como aconteceu em Singapura e nas Filipinas. 17

O gasto em saúde durante o primeiro ano de vida é muito menor quando as crianças estão em aleitamento materno de acordo com as recomendações internacionais. Nos EUA, resultados preliminares de um estudo de revisão sobre a factibilidade e o impacto de um programa de aleitamento materno numa Companhia de Seguros Médicos mostraram que o gasto com saúde de uma criança em aleitamento artificial, no primeiro ano de vida, é de aproximadamente 1146 dólares a mais, quando comparado com uma criança amamentada, especialmente em função dos gastos maiores por internações hospitalares. 17

A vantagem econômica do aleitamento materno é clara. Os resultados deste estudo mostram que prover a alimentação complementar da mãe carente, para que a mesma possa amamentar seu filho, além de ser mais saudável, tanto para a mãe, que melhorará seu estado nutricional, como para seu filho, que receberá o melhor alimento, o leite materno, traz vantagens econômicas importantes para famílias, unidades de saúde, estados, e governos. 17,19

\section{Conclusões}

O custo com a alimentação complementar da nutriz é cerca de $75 \%$ e $21 \%$ menor que o custo da alimentação do lactente com fórmula infantil e leite de vaca, respectivamente. Essa vantagem deve ser considerada, principalmente em famílias de baixo 
poder aquisitivo. Além disso, a qualidade de vida da nutriz melhor alimentada, das crianças amamentadas e de suas famílias tende a ser melhor, na medida em que há uma menor freqüência de morbidade, menos hospitalizações e maior intensidade do vínculo afetivo.

Os profissionais de saúde devem estar suficientemente conscientes desses benefícios, para que, no pré-natal e no pós-parto, possam orientar e apoiar às mães para que amamentem exclusivamente até os seis meses e continuem com a amamentação até os dois anos de idade ou mais.

Apesar das informações sobre a economia obtida com a amamentação serem importantes e úteis para os planejadores de saúde, como mais uma variável explicativa da relevância da amamentação, entendese que estes dados sobre valor econômico do aleitamento se agregam àqueles já sobejamente conhecidos, relativos à maior imunidade, fortalecimento do vínculo afetivo mãe e filho, e valor nutricional superior, entre outros.

Nesse sentido, é importante também que governos implantem políticas e programas que visem apoiar a complementação alimentar da nutriz de baixa renda, de forma a contribuir para a efetiva manutenção do período de amamentação recomendado, com o objetivo de melhorar os índices de aleitamento materno e reduzir a morbi-mortalidade infantil no país.

\section{Referências}

1. Giugliani ER. O aleitamento materno na prática clínica. J Pediatr 2000; 76 [Supl 3]: S238-S52.

2. WHO (World Health Organization). The optimal duration of exclusive breast feeding: results of a WHO systematic review. Geneva; The Organization; 2001.

3. Byers T, Graham S, Rzepka T, Marshall J. Lactation and breast cancer: evidence for a negative association in premenopausal women. Am J Epidemiol 1985; 121: 664-74.

4. Family Health International. Breast feeding as a family planning method. Lancet 1988; 2: 1204-5

5. OMS (Organização Mundial da Saúde). Evidencias científicas dos dez passos para o sucesso no aleitamento materno. Brasília (DF): A Organização; 2001.

6. Nóbrega FJ, Campos ALR. Distúrbios nutricionais e fraco vínculo mãe/filho. Rio de Janeiro: Revinter; 1996

7. Nurture Center to Prevent Childhood Malnutrition. The economic value of breast feeding: four perspectives for policymakers. Policy Series 1990; 1: 1-16.

8. Levine RE, Huffman SL. The economic value of breast feeding: a review of the literature. Washington (DC): Nurture Center to Prevent Childhood Malnutrition; 1990.

9. Ministério da Saúde. Secretaria de Políticas de Saúde, Organização Pan Americana de Saúde. Guia alimentar para crianças menores de dois anos. Brasília (DF); O Ministério; 2002.

10. Sanghvi TG. Melhora da eficácia em função dos custos da promoção do aleitamento materno em maternidades. New York: UNICEF; 1996.

11. Ministério da Saúde. Pesquisa de prevalência do aleitamento materno nas capitais e no Distrito Federal: relatório. Brasília (DF): O Ministério; 2001.

12. Araújo MFM. Situação e perspectivas do aleitamento materno no Brasil. In: Carvalho MR, Tamez RN. Ama- mentação: bases científicas para a prática profissional. Rio de Janeiro: Guanabara Koogan; 2002.

13. Araujo MFM, Del Fiaco A, Werner EH, Schmitz BAS. Incentivo ao aleitamento materno no Brasil: evolução do Projeto Carteiro Amigo da Amamentação de 1996 a 2002. Rev Bras Saúde Matern Infant 2003; 3: 195-204.

14. Ctenas ML, Vitolo MR. Crescendo com saúde: o guia de crescimento da criança. São Paulo: C2 Ed.; 1999.

15. OMS (Organização Mundial da Saúde). Necessidades de energia e proteína: São Paulo: Roca; 1998. (Série de Relatos Técnicos, 274).

16. Galeazzi MAM, Domeni SMA, Sichieri R. Estudo multicêntrico sobre consumo alimentar. Cad Debates 1997; [Especial].

17. WHO (World Health Organization). Wellstart International: Promoting breast feeding in health facilities: a short course for administrators and policy-makers. Genebra: The Organization; 1996. (Doc WHO/NUT/ 96.3).

18. OMS (Organização Mundial de Saúde), UNICEF (Fundo das Nações Unidas para a Infância). Aconselhamento em amamentação: um curso de treinamento: guia do treinador. São Paulo: Secretaria de Estado da Saúde; 1997.

19. WABA (World Alliance for Breastfeeding Action). World breastfeeding week. Available from: URL: http://www. waba.org.br/folder98.htm [2003 Sep 8]

20. Chetley A. The cost of bottle. Available from: URL: http://www.aleitammento.org.br/arquivos/bottle.htm [2003 Sep 08]

21. Cohen R, Mrtek MD, Mrtek RG. Comparision of maternal absenteeism an illness rates among breast feeding and formula-feeding women in two corporations. Am J Health Prom 1995; 10: 148-52.

Recebido em 14 de novembro de 2003

Versão final reapresentada em 04 de fevereiro de 2004

Aprovado em 15 de março de 2004 